\title{
Focus on behavioural genetics
}

\author{
European Journal of Human Genetics (2006) 14, 647-648. doi:10.1038/sj.ejhg.5201599
}

Few issues in genetic research attract more interest than how genes influence behaviour. The shades of eugenics, the promise of children chosen (or even engineered) for their behavioural constitution, hang over attempts to identify the genetic basis of mental illness, and particularly intelligence, with their seemingly inevitable appeals for controversy. And, at the same time, there stands one of the most challenging questions facing biology: how does a central nervous system, a brain that we know owes its constitution at least in part to the concerted action of a genome, give rise to behaviour?

Perhaps the most impressive advance in the last two decades is the acceptance that genetic approaches to this question are feasible. One of the standard objections, an excuse for the inadequacy of the field, used to be the supposed difficulty of the phenotype. Genetic studies of schizophrenia, for example, would fail because psychiatrists couldn't define the phenotype as well as endocrinologists could define diabetes. Second, even assuming there was progress on the phenotype issue, the molecular basis of behaviour was thought to be too complex as to defy dissection. A case in point is mental retardation where diagnosis is often straightforward but aetiology hardly progressed beyond classifying causes into pre-, peri- and post-natal. A spectrum of physical abnormalities such as facial dysmorphism, short stature and minor physical anomalies would point unambiguously to a biological, almost certainly genetic cause in many cases, but the undoubted heterogeneity of the condition appeared to make finding those genes an impossible proposition. Third, in the extremely unlikely event that genes could be found, our ability to carry out functional investigation would be so restricted as to make gene identification of limited interest. This was for two reasons. First, without a cellular or other in vitro assay, gene function could only be tested in whole animal experiments. Behavioural assays in mice (the model organism of choice) were primitive and were likely to be prone to the same drawbacks as human phenotypes. Second, there were many aspects of gene function that simply could not be modelled in animals, language for example.

The collection of articles in this issue is one illustration of the extent to which progress has been made. Psychiatric illnesses have become fair game for geneticists, almost mainstream interests. Questions about the accuracy and reliability of diagnoses have rather surprisingly turned into questions about the relationship between phenotypic features based on known, or inferred, genetic similarity. This is not to say that phenotypic definitions for genetic analysis are now agreed upon, or that the extent of the difficulty has been exaggerated; far from it. As the articles on mood disorders, schizophrenia and developmental dyslexia show, diagnosis remains a key concern. There is an acceptance that a single diagnostic categorization is probably not obtainable (as Riley and Kendler ${ }^{1}$ point out in their discussion of schizophrenia, it is 'common to perform several analyses of data using a number of different definitions of illness') but also a realization that genetic data can be used to inform the diagnostic process itself. Craddock and Forty ${ }^{2}$ make this point strongly: 'Molecular genetic findings are likely to catalyze a re-appraisal of psychiatric nosology'. They argue that genetic data challenge the orthodox separation of schizophrenia from mood disorders, in other words that there may be a common genetic vulnerability to both conditions. That genetic action in behaviour is complex, cutting across accepted diagnostic categories, has been known for some time from quantitative genetics (for example in the genetic susceptibility to some anxiety disorders and depression). ${ }^{3-5}$ The novelty is that this might be observed at the level of a locus, or of a single variant in a gene: some genetic variants contribute to the one disorder, some to another and yet others to both.

There is little doubt that the genetic basis of behaviour is complicated, so much so that it continues to defy molecular dissection: gene finding remains frustratingly difficult in psychiatric disorders. Nevertheless there is no doubt that molecular approaches have been extraordinarily, and unexpectedly, successful in identifying mutations that give rise to mental retardation. Chelly et al. ${ }^{6}$ explain the impact of their work, demonstrating that even the rarest monogenic condition can be tackled and can deliver important new insights into pathophysiology. The story is strikingly similar to the hunt for genetic factors that contribute to diabetes $^{7}$ and hypertension ${ }^{8,9}$ where the investigation of rare monogenic conditions has been critical in unveiling molecular mechanisms, and also suggesting candidate genes that contribute to risk in the general population. Genetic studies of intelligence, as Deary et al. ${ }^{10}$ make clear, has not, yet, benefited from the 
molecular insights gleaned from monogenic forms of mental retardation, but evolutionary biology has. Lahn and co-workers, starting with genes identified from analysis of patients with severe reductions in cerebral cortical size and mental retardation, have found evidence that these genes experienced strong adaptive evolution in the descent of Homo sapiens, consistent with their role in the evolutionary development of the human brain. ${ }^{11,12}$

Advances in genome biology have made functional analysis of all mammalian genes a feasible proposition, even for genes that influence behaviour. Godinho and Nolan ${ }^{13}$ describe the application of mutagenesis in the mouse for the creation of behavioural mutants. They explain how many of the same issues of obtaining robust phenotypes confront mouse behavioural geneticists. Nevertheless, the advantages of using a model system are immense: mouse mutants are an essential tool for progress in explaining the origins of behaviour. Mouse genetics can also be complex, as Willis-Owen and Flint ${ }^{14}$ explain, but still tractable: genetic approaches to complex quantitative phenotypes are poised to make a significant contribution to our understanding of the molecular basis of those conditions which can be modelled in mice.

Mice are not the only model organism that inform studies of human behaviour. Rosato et al. ${ }^{15}$ pointing to the pervasive importance of circadian rhythms on behaviour, describe what studies in Drosophila have revealed. Remarkably, mutations in a human period gene, the closest homolog to that in the fly, make their carriers go to bed early and get up early, just as happens in the mutant invertebrate. Here is one example where molecular insights gained from flies, humans and mice have all contributed to our understanding of a basic biological process. No doubt similar advances will continue to emerge from the coordinated efforts of geneticists working from very different angles on the origins of behaviour.

\author{
Jonathan Flint ${ }^{1}$ and Anthony P. Monaco ${ }^{1}$ \\ ${ }^{1}$ Wellcome Trust Centre for Human Genetics, Roosevelt Drive, \\ Oxford OX3 $7 B N$, UK
}

\section{References}

1 Riley B, Kendler KS: Molecular genetic studies of schizophrenia. Eur J Hum Genet 2006; 14: 669-680.

2 Craddock N, Forty L: Genetics of affective (mood) disorders. Eur J Hum Genet 2006; 14: 660-668.

3 Kendler KS, Neale MC, Kessler RC, Heath AC, Eaves LJ: Major depression and generalized anxiety disorder. Same genes, (partly) different environments? Arch Gen Psychiatry 1992; 49: 716-722.

4 Roy M-A, Neale MC, Pedersen NL, Mathé AA, Kendler KS: A twin study of generalized anxiety disorder and major depression. Psychological Medicine 1995; 25: 1037-1049.

5 Kendler KS, Neale MC, Kessler RC, Heath AC, Eaves LJ: Major depression and phobias: the genetic and environmental sources of comorbidity. Psychological Medicine 1993; 23: 361-371.

6 Chelly J, Khelfaoui M, Francis F, Chérif B, Bienvenu T: Genetics and pathophysiology of mental retardation. Eur J Hum Genet 2006; 14: 701-713

7 O'Rahilly S, Barroso I, Wareham NJ: Genetic factors in type 2 diabetes: the end of the beginning? Science 2005; 307: 370-373.

8 Lifton RP, Gharavi AG, Geller DS: Molecular mechanisms of human hypertension. Cell 2001; 104: 545-556.

9 Lifton RP, Wilson FH, Choate KA, Geller DS: Salt and blood pressure: new insight from human genetic studies. Cold Spring Harb Symp Quant Biol 2002; 67: 445-450.

10 Deary IJ, Spinath FM, Bates TC: Genetics of intelligence. Eur J Hum Genet 2006; 14: 690-700.

11 Evans PD, Gilbert SL, Mekel-Bobrov N et al: Microcephalin, a gene regulating brain size, continues to evolve adaptively in humans. Science 2005; 309: 1717-1720.

12 Mekel-Bobrov N, Gilbert SL, Evans PD et al: Ongoing adaptive evolution of ASPM, a brain size determinant in Homo sapiens. Science 2005; 309: 1720-1722.

13 Godinho SIH, Nolan PM: The role of mutagenesis in defining genes in behaviour. Eur J Hum Genet 2006; 14: 651-659.

14 Willis-Owen SAG, Flint J: The genetic basis of emotional behaviour in mice. Eur J Hum Genet 2006; 14: 721-728.

15 Rosato E, Tauber E, Kyriacou CP: Molecular genetics of the fruitfly circadian clock. Eur J Hum Genet 2006; 14: 729-738. 